framework has been addressed in a recent and very provocative paper by Buston and Emlen (2003). They suggest that mate choice, at least among Cornell undergraduates, is not governed by mate "potential" attributes - for example, attractive women choosing strong, high-status males, and strong, high status men choosing attractive women. Instead, they found that individuals choices were based on their perceptions of the possible duration of the relationships. When there was expectation of a long term, stable relationship, individuals chose mates similar to themselves (on factors such as physical attractiveness, status, and commitment to family). Attractive women chose attractive men; they did not choose strong, aggressive men. Situations in which costs associated with mate switching is high, for example, where divorce is economically disruptive, would result in individuals choosing mates who are similar to themselves (see Borgerhoff Mulder 2004).

By contrast, aggressive men (again, where aggression is intrasexual) were chosen by physically attractive women in situations in which the quality of available mates is low. In this latter case, male-male aggression would be reinforced by female choice and females would "sample" widely from available males for a strong mate who also protects and provisions her and her offspring.

With these limitations stated, Archer's target article makes an important contribution toward the theoretical integration of a wide and disparate literature. He has done the field an important service.

\section{There's no contest: Human sex differences are sexually selected}

\section{doi:10.1017/S0140525X0999032X}

\section{Nicholas Pound, ${ }^{\mathrm{a}}$ Martin Daly, ${ }^{\mathrm{b}}$ and Margo Wilson ${ }^{\mathrm{b}}$ \\ ${ }^{a}$ Department of Psychology, School of Social Sciences, Brunel University, Uxbridge UB8 3PH, United Kingdom; ${ }^{\mathrm{b}}$ Department of Psychology, Neuroscience, and Behaviour (PNB), McMaster University, Hamilton, Ontario L8S 4K1, Canada. \\ nicholas.pound@brunel.ac.uk \\ daly@mcmaster.ca \\ wilson@mcmaster.ca}

Abstract: An evolutionary psychological perspective drawing on sexual selection theory can better explain sex differences in aggression and violence than can social constructionist theories. Moreover, there is accumulating evidence that, in accordance with predictions derived from sexual selection theory, men modulate their willingness to engage in risky and violent confrontations in response to cues to fitness variance and future prospects.

In the target article, Archer argues persuasively that an evolutionary psychological perspective drawing on sexual selection theory can account for observed sex differences in aggression and violence more parsimoniously than social constructionist theories. In our view, however, the case for sexual selection's role in the evolution of these sex differences is even stronger than Archer's treatment suggests, and he concedes too much to advocates of the discredited null hypothesis that female and male psyches are undifferentiated.

According to Archer (sect. 2.2.1), the "main alternative" to a selectionist explanation of the origins of the sex differences of interest is the "social role" theory of Eagly and Wood (1999; Wood \& Eagly 2002). At best, these authors can be read as offering a partial account of ontogenetic processes in sexual differentiation, which, if upheld, would complement an evolutionary account. At worst, they can be read as proposing that the only evolved differences between women and men are "physical" (i.e., non-neural anatomical differences), and if this is indeed their meaning, they are simply uninformed (see, e.g., Kimura
1999). In neither case have they provided a viable "alternative" to an account that gives centre stage to sexual selection.

In explaining why sexual selection should have made men more intensely competitive than women, Archer (sect. 3.2) aptly cites anatomical, demographic, and behavioural evidence that Homo sapiens evolved as an effectively polygynous species in which male fitness variance exceeded female fitness variance. Recent genetic evidence (Wilder et al. 2004) reinforces this conclusion: In both our species as a whole and in discrete subpopulations thereof, the most recent common ancestor (MRCA) of mitochondrial DNA, inherited matrilineally, lived about twice as long ago as the MRCA of Y chromosomes, inherited patrilineally. These results provide strong evidence that individual men have consistently faced a higher risk of reproductive failure than individual women.

Archer notes (sect. 2.8) that, consistent with sexual selection theory, there is evidence that males with limited access to reproductive opportunities, or the resources required to obtain such opportunities, are more likely to resort to violence. However, there is accumulating evidence to support a more specific prediction derived from the same theoretical perspective, namely that the prevalence of dangerous confrontations should vary predictably according to variations in the local intensity of intrasexual competition and that cues to higher fitness variance should lead males to modulate their willingness to engage in risky and violent interactions with other men (for review, see Wilson et al. 2002; 2009).

In an effectively polygynous species, the intensity of male-male competition will in part depend on the extent to which the resources required to obtain reproductive opportunities are distributed equitably. Extreme inequity creates a situation where those at the bottom have little to lose if they escalate their tactics of competition, and much to gain. Consequently, cues to inequity should lead to facultative adjustments in men's willingness to employ violent and risky tactics to gain status and resources, which are the means to fitness. Consistent with this, evidence indicates that relative deprivation (as indexed by income inequality) is typically a more powerful predictor of variation in male violence than other socioeconomic measures such as percent below the poverty line or average income (Daly \& Wilson 2001). In both cross-national and more local comparisons, the Gini index of income inequality consistently outperforms most other socioeconomic predictors of homicide rates (e.g. Blau \& Blau 1982; Daly et al. 2001).

Increased willingness to resort to violence where resources are distributed inequitably is not uniquely predicted by sexual selection theory. However, in contrast to social constructionist accounts, an evolutionary psychological perspective treats such increased risk-proneness as a facultative adaptive response to situations where the distribution of resources is such that excessive risk-aversion will lead to substantially reduced expected fitness (Wilson et al. 2002; 2009).

An evolutionary psychological approach based on sexual selection and life history theory also predicts that individuals should modulate their willingness to engage in risky and violent confrontations according to cues of future survival and hence reproductive prospects - in other words, when prospects are poor, organisms may be expected to discount the future steeply in the pursuit of more immediate goals (Daly \& Wilson 2005). Archer notes (sect. 3.1) that greater male than female mortality is characteristic of the sexually selected "adaptive complex" generated by intense inter-male competition, but the target article could perhaps have examined the implications of this in more detail. It is not just that males are likely to discount the future more heavily than females as a consequence of the sex difference in mortality; moreover, future discounting and willingness to engage in risky escalation of social conflicts are expected to vary predictably in relation to future survival prospects. Where these are poor, men should become particularly risk prone and willing to risk death in violent altercations as they compete for 
the resources required to obtain reproductive opportunities, and directly for the opportunities themselves. Consistent with this, Wilson and Daly (1997) found that across neighbourhoods in a major U.S. city (Chicago), the best statistical predictor of homicide rates was low male life expectancy (even with homicide as a cause of death removed).

Finally, we do not understand Archer's rationale for suggesting that a sexual selection approach warrants the "prediction" that the sexes will not differ in "aggression" or "anger," but only in how they manifest these things. Sell's research and theorizing (e.g., Sell et al. 2005), which Archer cites, clearly suggests that insofar as becoming angry entails an elevated risk of violent confrontation, we may expect people to differ adaptively in their readiness to anger. Why should this proposition not apply to sex differences? More fundamentally, what does it even mean to suggest that men and women do not differ in "aggression" or "anger," but only in the manifestations thereof? We lack both consensual definitions and good metrics of these states, and finding that the sexes give the same mean answer on a selfreport scale of "aggression" or "anger" is uninformative. Consequently, evidence for the popular claim that men and women are equally aggressive, but that the former manifest their aggression "directly" (e.g., as violence) and the latter "indirectly" (e.g., as innuendo) is not convincing.

\section{Sex differences in dream aggression}

doi:10.1017/S0140525X09990306

\section{Michael Schredl \\ Sleep Laboratory, Central Institute of Mental Health, J5, 68159 Mannheim, Germany. \\ Michael.Schredl@zi-mannheim.de \\ www.dreamresearch.de}

Abstract: Dream research shows sex differences in dream aggression that fit very well with the findings for waking-life aggressive behaviour. Dream studies are a valuable tool for investigating variables underlying the sex difference in aggression. One might argue that studying dream aggression might be even more promising because aggression in dreams is not socially labelled, as being aggressive in waking life is.

Since dreams reflect waking life experiences (the so-called continuity hypothesis of dreaming; Schredl 2003), dream studies can elucidate sex differences reported for waking behaviour. For example, it is a stable finding that men report more sexual dreams than do women (Schredl et al. 1998), which reflects the meta-analytic findings of higher frequency of masturbation and sexual fantasies in males compared to females (Oliver \& Hyde 1993). Regarding aggression in dreams, the findings are in line with the meta-analysis reported by Archer: Men's dreams included more physical aggression than women's dreams did (Hall \& Van de Castle 1966), whereas the amount of verbal aggression did not differ between the sexes (Schredl et al. 1998). The gender difference regarding the percentage of physical aggression (50\% in men's dreams vs. 34\% in women's dreams; Hall \& Van de Castle 1966) is quite stable over time. The data collection period of Hall and Van de Castle (1966) ranged from the late 1940 s to the early '50s. Subsequent studies in the '70s (Hall et al. 1982) and '90s (Schredl et al. 2003), and very recent studies (Schredl \& Keller 2008-2009) replicated the higher prevalence of physical aggression in men's dreams compared with total aggression. This means that cultural developments such as the women's movement did not affect this sex difference in dreams. And this favours the sexual selection theory over the social role theory.
Another aspect of dream aggression fits the theory put forward by Archer: the higher difference in the aggression per male character compared to the aggression per female character in men ( 0.23 vs. 0.13 for men, 0.13 vs. 0.10 for women). That is, women experience aggressive interactions with both men and women in almost equal frequency in their dreams, whereas men's dreams include same-sex aggression more often compared to opposite-sex aggression (Hall \& Domhoff 1963).

Also very interesting is the shift in the percentage of physical aggression over the life-span. Whereas children (younger than 11 years old) showed the typical gender difference of more physical aggression in boy's dreams compared with girl's dreams, the ratio of physical aggression in dreams is the same for both sexes in the age range from 12 to 17 years (Hall \& Domhoff 1963; Oberst et al. 2005). This fits with Archer's argument that males avoid risky encounters with older males prior to adulthood. The adults - as reported above - again showed the preponderance of physical aggression in men's dreams.

Domhoff (1996) reviews cross-cultural dreams studies. Whereas many Western countries showed higher prevalence rates of dream aggression in men compared to women, there where several exceptions. The Hopi Indians, for example, showed no gender differences in overall aggression and in the percentage of physical aggression (Domhoff 1996). The term Hopi can be translated into "peaceful ones" reflecting the lifestyle of these Pueblo Native Americans. But for some industrial countries, such as Switzerland and Japan, the ratios of physical dream aggression were not different between the sexes. This indicates that cultural factors modulate the amount of aggression in dream. In females, dream aggression was more often found in dreams of non-traditional women, indicating again the cultural effect on aggression pointed out by Archer. Unfortunately, these studies did not differentiate between same-sex aggression and opposite-sex aggression to enable us to test Archer's claim that cultural factors might be more important in explaining the amount of opposite-sex aggression.

Two studies, by Waterman et al. (1988) and Cohen (1973), investigated whether biological sex or feminine versus masculine sex role orientation explains differences in dream aggression. Whereas the finding of the first study was unambiguous (only biological sex was of importance), the second study showed effects of both variables on the amount of dream aggression. Again, it would have been fruitful to differentiate between same-sex and opposite-sex aggression.

Another interesting gender difference can be found in the bad dreams and nightmares of children (Schredl \& Pallmer 1998). In Table 1, the percentages of male and female aggressors are presented.

Male characters threaten the dreamer most often whereas women are quite rarely aggressors in dreams. The ratio of male and female aggressors in dreams is similar for boys and girls, thus reflecting the preponderance of male aggression in mass media (news, films, etc.). It would be very interesting to study the gender of the aggressor in cross-cultural dream studies in more detail, for example, in societies without predominance of male aggression. If dream aggressors are still mostly male, one

Table 1 (Schredl). Human aggressors in children's dreams

\begin{tabular}{llll}
\hline \hline Aggressors & $\begin{array}{l}\text { Total } \\
(\mathrm{n}=111)\end{array}$ & $\begin{array}{l}\text { Boys } \\
(\mathrm{n}=35)\end{array}$ & \multicolumn{1}{l}{$\begin{array}{l}\text { Girls } \\
(\mathrm{n}=76)\end{array}$} \\
\hline Male (unfamiliar) & $49.7 \%$ & $51.4 \%$ & $48.7 \%$ \\
Female (unfamiliar) & $3.6 \%$ & $0.0 \%$ & $5.3 \%$ \\
Male (familiar) & $27.8 \%$ & $31.4 \%$ & $26.2 \%$ \\
Female (familiar) & $18.9 \%$ & $17.2 \%$ & $19.8 \%$ \\
\hline \hline
\end{tabular}

